\title{
Automatic Lung Lobe Segmentation and Fissure Extraction Using Itempls Method on Computer Tomography Images
}

\author{
K.K.Thanammal ${ }^{1}$ and J. S. Jayasudha ${ }^{2}$ \\ ${ }^{1}$ Associate Professor, Department of MCA \\ S.T.Hindu College, Nagercoil \\ ${ }^{2}$ H.O.D, Computer Science and Engineering \\ Sree Chitra Tirunal College of Engineering, Tiruvananthapuram \\ thanaravindran@gmail.com ${ }^{1}$, jayasudhajs@gmail.com ${ }^{2}$
}

\begin{abstract}
This paper proposes an automatic lung lobe segmentation method in a supervised way. The lung image is given as input and the lung image is segmented using iterative threshold method. Then fissure information is enhanced using log supportive filter. Then Fissure locations are extracted using Vector training and matching process. Finally the lung lobes are segmented and marked as individual lobes. The main modules of this proposed method are Iterative threshold based lung segmentation, Fissure enhancement, fissure detection and Lung lobe segmentation. This proposed method can be used to find the missing fissures and unclear fissures also. This proposed method is working based on training of fissure information and detection is done according to the trained vectors. The proposed method segments the lung lobes with high performance and high speed.
\end{abstract}

Keywords: Lung lobes, CT image, fissures.

\section{Introduction}

The human lungs are subdivided into five lobes that are separated by visceral pleura called pulmonary fissure. There are three lobes in the right lung, namely upper, middle, and lower lobe. The right upper and right middle lobes are divided by the right minor fissure whereas the right major fissure delimits the lower lobe from the rest of the lung. The segmentation of pulmonary lobes is challenging because of anatomical variation and incomplete fissures. On the one hand, pathologies can deform the lobes and make the fissures unrecognizable. And on the other hand, even in patients with normal lung parenchyma the fissures are often not complete. Lung lobe segmentation is relevant in clinical applications particularly for treatment planning. The location and distribution of pulmonary diseases are important parameters for the selection of a suitable treatment. The anatomy of lung is illustrated in figure 1. Computed tomography (CT) is the most widely used imaging technology for diagnosing and treating lung diseases, the third leading cause of death in North America [1]. Modem CT scanners produce a stack of about 500 isotropic images ( $0.6 \mathrm{~mm}$ in thickness) for a complete scan of an average patient's lung cavity. Current clinical protocol combines these 500 isotropic images into approximately 60 clinical images, by merging several isotropic images into a single clinical image. Reading clinical images result in loss of valuable information, which isotropic CT Images provide. 


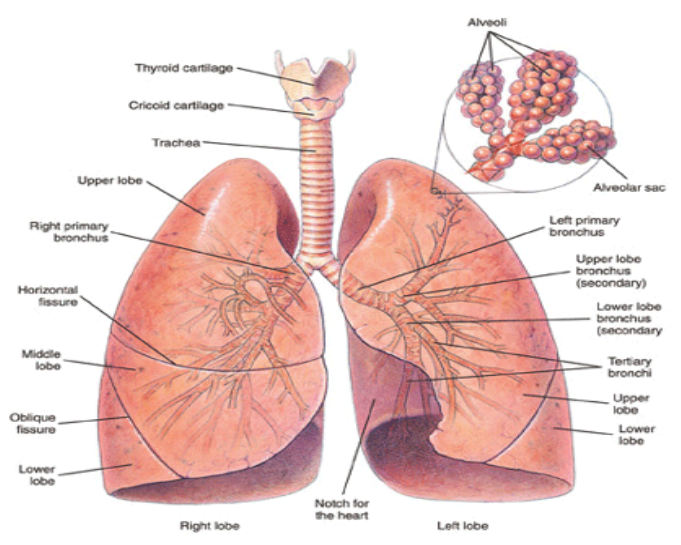

Figure 1. Anatomy of Lung

The alleviation of this situation needs an efficient diagnostic/treatment system employing isotropic images. For this reason, we focus on developing an approach of segmenting lung lobes from isotropic CT images (referred to as CT images from here on in). Recent advances in virtual reality technologies have made three-dimensional (3D) visualization of lungs feasible for diagnosing and treating lung diseases [2-4]. Compared to 2D grayscale CT images, 3D visualization of lungs provide multi-viewpoints, colors, stereoscopic views, and no need of mental reconstruction. Our previous study has revealed that $3 \mathrm{D}$ visualization of lungs can significantly reduce surgeon's workload and planning time, as well increase the accuracy of lung surgeries [2]. Typical human lungs consist of 5 lobes, separated by 3 fissures (boundaries of lung lobes). The left oblique fissure (LOF) divides the left lung into two lobes: the superior and inferior lobes; while the right oblique fissure (ROF) and the right horizontal fissure (RHF) separate the right lung into three lobes: the superior, middle and inferior lobes. In 2D CT images, fissures appear as curvilinear lines on each CT image [5]. In a stack of CT images, these fissures from 3D surfaces separating adjacent lung lobes. Complete segmentation of the lung lobes by automatically identifying fissure surfaces-3D surfaces formed by fissures in individual CT images-is the foremost requirement of achieving 3D visualization of lungs. Automatic identification of fissure surfaces is a non-trivial task due to the fissures' variable shape and appearance, along with the low contrast and high noise inherited in CT images [5]. As well, identification of the fissures on individual 2D CT images does not always produce complete segmentation of the lung lobes. Studies have a shown that approximately $70 \%$ of fissures are incomplete and it is impossible to observe them even by an expert [6, 7]. Furthermore, lung diseases may disrupt and deform fissures causing discontinuous between fissures in adjacent CT images. Traditional segmentation methods such as deformable models [8] and level sets [9] are unsuitable for fissure identification due to CT image noise, incomplete fissure, and the need for manual initialization. Thus, complete segmentation of diseased lung lobes remains an arduous task.

\section{Methodology}

The main objective of this paper is to segment the lung lobes for surgical preplanning, which is important to human lung medical treatments. It is implemented using MATLAB (10.0) and lung segmentation is preformed. The input CT scan lung images are taken from LOLA11 lung data set. The lung image must be in $256 \times 256$ dimensions and it should be an 8 -bit image. ITEMPLS method is iterative threshold and euclidean matching pulmonary lobe 
segmentation method. The following block diagram 2 illustrates the proposed method in detail.

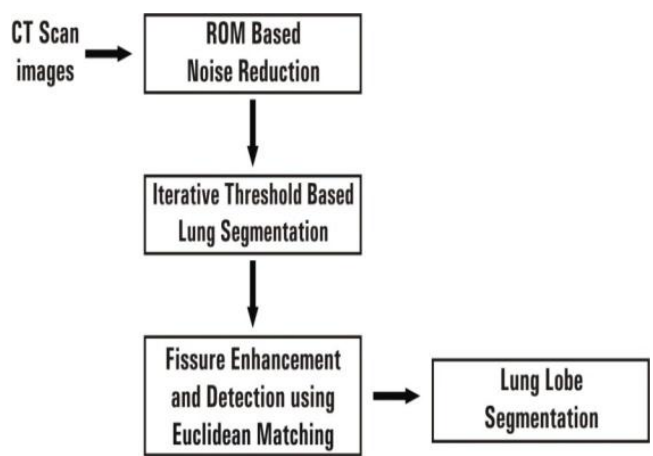

Figure 2. ITEMPLS Block Diagram

The above diagram 1 discusses the implementation details of iterative threshold and Euclidean matching method.

\subsection{Iterative Threshold Based Lung Segmentation}

In the proposed method, the noise reduction is done using ROM filter. ROM filter is Rank Order Median Filter. The lung segmentation is done on filtered image. Iterative threshold method is applied in this paper to segment the lung. Segmentation is often considered to be the first step in image analysis. It is hope that the regions obtained correspond to the anatomical information of a lung image (3-D) representation by the image (2-D). Image segmentation methods assume that the intensity values are different in different regions [10]. Thresholding classifies pixels into two categories, those to which some property measured from the image falls below a threshold, and those at which the property equals or exceeds a threshold. Thresholding creates a binary image this is a binarization process. The segmentation process of the iterative threshold segmentation can be described as follow

Input: Noise free lung image

Output: Lung segmented image.

Step 1: $\quad$ The lung pixel information is stored as the 2-Dimensional matrix.

Step 2: $\quad$ Threshold initialization is done through the formula is Threshold initialization $=\operatorname{Max}($ Max $($ Lung_image $)) / 2$.

Step 3: $\quad$ Assign threshold value equal to initial threshold value.

Step 4: $\quad$ Mark the pixels which are greater than initial threshold as 255 and mark the pixels which are less than threshold as 0 in flag array.

$$
\begin{gathered}
\text { if lung_image }(i, j)>\text { threshold } \\
\text { flagarray }(i, j)=255 \\
\text { else } \quad \text { flagarray }(i, j)=0
\end{gathered}
$$

Step 5: $\quad$ Find the lung image intensities which are having flag array values 255 and sum them. Also find the number of pixels which are undergone this summing process.

Step 6: $\quad$ Find the lung image intensities which are having flag array values zero and sum them. Also find the number of pixels which are undergone this summing process. 
Step 7: $\quad$ Find the mean of the above threshold pixels using summed up value and the pixel count of 255 marked flag array.

Step 8: $\quad$ Find the mean of the below threshold pixels using summed up value and the pixel count of zero marked flag array.

Step 9: $\quad$ Now find the next iteration threshold as

Threshold $=($ Mean of above threshold pixels +

Mean of below threshold pixels) / 2 .

Step10: $\quad$ Do the steps from steps 5 to 10 for six time iteration.

Step11: Convert the flag array content as a binaries one which is having zeros and ones.

Step12: $\quad$ Find the individual parts of the binary image using bwlabel command of matlab, this is known as labeled image.

Step13: $\quad$ Fill the image region and holes using imfill command of matlab.

Step14: $\quad$ Find the absolute difference of the labeled image and the filled image. Separated_lungs $=$ abs (labeled_image- filled_image).

\subsection{Refinement Process of Lung Segmentation}

The refinement process remove some unwanted information from the lung images such as blood vessels, air holes, noise and also orphan pixels are removed using the morphological operation. Morphological dilation operation is used to filling in small air holes. Dilation adds pixels to the boundaries of objects in an image. The value of the output pixel is the maximum value of all the pixels in the input pixel's neighborhood. The number of pixels added from the objects in an image depends on the size and shape of the structuring element used to process the image. Dilation operator used the diamond structuring element value four. Morphological erosion operator is used to eliminating small protrusion and orphan pixels from lung boundaries. Erosion removes pixels on object boundaries. The value of the output pixel is the minimum value of all the pixels in the input pixel's neighborhood. The number of pixels removed from the objects in an image depends on the size and shape of the structuring element used to process the image. Erosion operator used the diamond structuring element value as four. Morphological thinning operation that is used to remove selected foreground pixels from binarized image that is background information is removed from lung image. Finally separated lung images are preserved.

\subsection{Lung Fissure Enhancement Process}

The lung fissure enhancement process enhanced the lung fissure. The enhanced fissure is more suitable than the original lung fissure image and log transform method is used. In log transformations compress the dynamic range of separated lung image with large variations in pixel values. This process also suppressed some unwanted information such as blood vessels, air holes, cancerous parts and noisy contents. Image enhance approaches fall into two broad categories as spatial domain and frequency domain. Fissure enhancement process used logarithmic values as

$$
\text { Enhanced_image }=\log _{10} \text { (projected image) }
$$

Next step is find the maximum value of the enhanced image and range fitting process used as 
Final_enhanced_image $=($ Enhanced_image $/$ Max value $) \times 255$

In range fitting process set the range as, remove the value is greater than 175 and set that location on zero. This will remove the unwanted higher value range such as blood vessels and cancer parts that are remove the value less than 50 and set that location on zero. This will remove the unwanted low range values which are not being in the fissure supported range.

To extended fissure range using the formula as

$$
\text { Enhanced_fissure_Image }=(\text { Fissure _Range _Image }-50) \times 2
$$

Finally enhanced fissure part used as following formula

Final fissure_enhanced_image $=($ Redistributed_image + Non-redistributed image $) / 2$

It provide mean value of image redistributed and non-redistributed in the fissure enhanced image.

\subsection{Training Process}

The training process trains the fissure information from the training set lung images. The training set lung images are decided and the ground truth of fissure information is marked by human. The training process read the enhanced lung image and ground truth fissure image. Find land marks (Fissure content) to match across images and mapping between image areas may be non-linear or complicated. Some time error may be occurred in the location of detected points. To consider enhanced image pixel information for detected fissure locations and enhanced image pixels are projected on detected fissure parts are maintained as trained image for training purpose.

\subsection{Query Lung Segmentation and Enhancement}

User chosen the query lung image and the lung image pixel information are stored in memory and iterative threshold method is applied to separate the lung images into a binarized one. The segmented lungs are refined using the morphological operation some unwanted information is removed such as blood vessels, air holes, cancer parts and noise. Log based method used to enhance the fissure part. The required regions of fissure parts are enhanced and get visually clear fissure content.

\subsection{Euclidean Distance Based Fissure Extraction}

Fissure extraction process is done by the test process. These test process considering with the training process. The trained vector is used to match the query fissure. The Euclidean matching process is done for testing process [11]. Fissure extraction process based on the threshold value. Euclidean distance based fissure extraction process set the block size as 3 that is distance matrix. Select block query image surrounded by $3 \times 3$ window which have center point as fissure enhanced image foreground pixel. Select the fissure trained image and also select block training image from the fissure training image. Calculate Euclidean distance value from for the Block Query and Block Training using the following formula for the fissure marked pixels 


$$
\sum_{i=0, j=0}^{i=2, j=2}(\text { BlockQuery }(i, j)-\text { BlockTraining }(i, j))^{2}
$$

Euclidean distance sum value is less than 100 then the fissure detection output is marked and set to 255. Repeat this process as all foreground pixels of fissure enhanced image of query lung. Query lung fissure detected image is displayed in figure window.

\subsection{Refinement Process of Fissure}

The refinement process of lung segmentation is like that the refinement process of fissure. The refinement process removes some unwanted information from the lung fissure using the morphological operation. Morphological dilation operation is used to filling in small air holes. Dilation operator used the diamond structuring element value four [12]. Morphological erosion operator is used to eliminating small protrusion and orphan pixels from lung boundaries. Erosion operator used the diamond structuring element value as four. Morphological thinning operation that is used to remove selected foreground pixels from binarized image that is background information is removed from lung image. Final fissure part is find out using the formula as

Fissure image $=$ abs (partially detected fissure - non fissure image $)$

\subsection{Lung Lobe Segmentation Using Region Filling}

Region filling is the process which is used to fill the irregular shaped regions with a solid color which is having the high similarity intensity values [12]. The Flood Fill algorithm is a recursive graphics algorithm used to fill in irregular shaped regions with a solid color. Some complicated regions filling are taken the scan line approach and coloring the interior of an irregular region. The left lung lobes are filled using Flood Fill with different color information. The right lung lobes are filled using Flood Fill with different color information. The fissures are projected into the lung image and displayed segmented lung lobes.

\section{Conclusion}

This paper presented a lobe segmentation method that detects fissure and segments lobes. The approach is anatomically inspired and similar to the way humans determine the lobar boundary. Visible fissures are used for segmentation because they are the most precise feature. Three main components namely Lung segmentation, Fissure enhancement and lung lobe segmentation are working without error and produce better outputs. The vector matching based lung lobe segmentation detects the fissure at higher level. It is better than the existing lung lobe segmentation methods in the case of segmentation accuracy measurement such as MSE and PSNR. This paper presented a fast automatic lobar segmentation method and performs well and is robust against missing fissures. 


\section{Experimental Results}

Original query noisy lung image

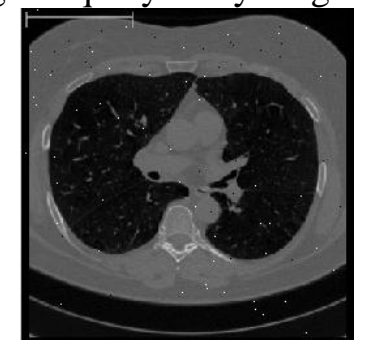

Lungs segmentation using iterative threshold method

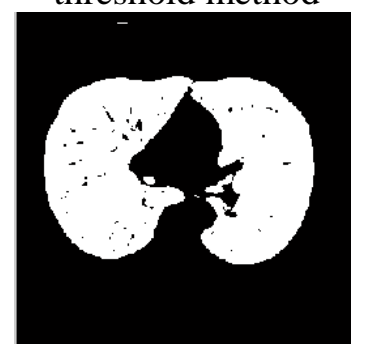

Enhanced fissure and blood vessel removed image
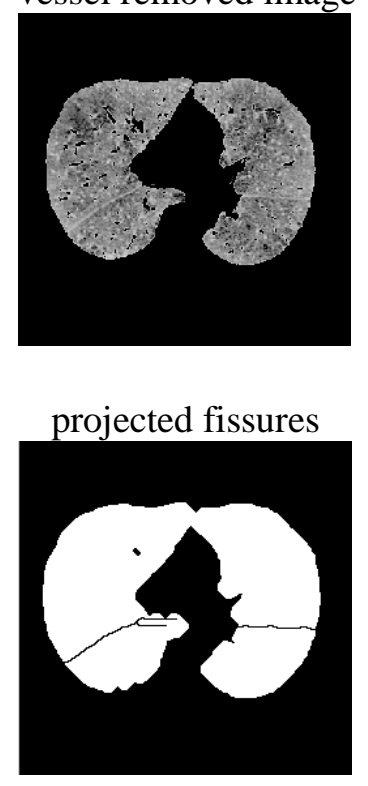

Rank order median filter result

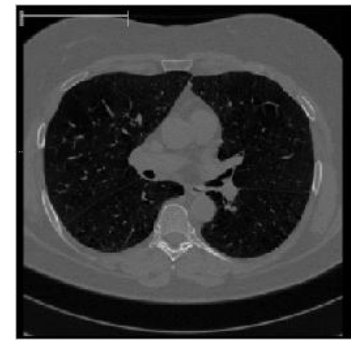

Final lung segmentation

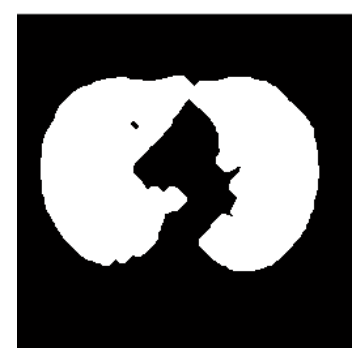

Fissure detection using vector matching
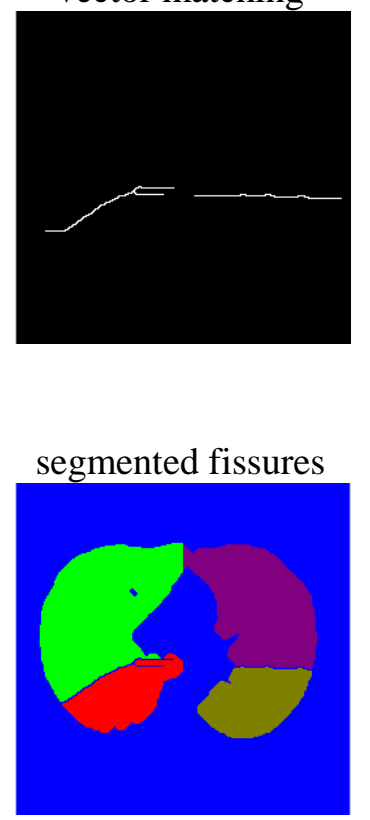

Figure 4. Screens for ITEMPLS Method

\section{References}

[1] American Cancer Society, Cancer Facts and Figures 2008.Atlanta: American Cancer Society, (2008). 
[2] Y. Hu and R. A. Malthaner, "The feasibility of three-dimensional displays of the thorax for preoperative in the surgical treatment of lung cancer,"Europ. Cardio-Thoraic Surg., vol. 31, (2007) pp. 506-511.

[3] J-M. Kuhnigk, V. Dicken, S. Zidowitz, L. Bornemann, B. Kuemmerlen, S. Krass, H-O. Peitgen, S. Yuval, HH. Fend, W. S. Rau, and T. Achenbach, "Newtools for computer assistance in thoracic CT.Part 1. Functional analysis of lungs, ling lobes, and bronchopulmonary segments,"RadioGraphics, vol. 25, (2005), pp. 525-536.

[4] B. M. Hemminger, P. L. Molina, T. M. Egan, F. C. Detterbeck, K. E. Muller, C. S. Coffrey, and J. K. Lee, "Assessment of real-time 3D visualization for cardiothoracic diagnostic evaluation and surgery planning", J. Digit. Imag., vol. 18, (2005), pp. 145-153.

[5] W. R. Webb, N. L. Muller, and D. P. Naidich, "High-resolution CT of the hung", $3^{\text {rd }}$ ed., Philadelphia, PA: Lippincott, Williams and Wilkins, (2001).

[6] K. Hayashi, A. Aziz, K. Ashizwa, H. Hayashi, K. Nagaoki, and H. Otsuji, "Radiographic and CT appearances of the major fissures", Radiographics, vol. 21, (2001), pp. 861-874.

[7] B. N. Raasch, E. W. Carsky, E. J. Lane, J. P. O' Callaghan, and E. R. Heitzman, "Radiographic anatomy of the interlobar fissures: a study of 100 specimens", AJR, vol. 138, (1982), pp. 647-554.

[8] M. Kass, A. Witkin and D. Terzopoulos, "Snakes: active contour models," Int J. Comp. Vis., vol. vl, (1987), pp. 321-331.

[9] R. Malladi, J. A. Sethian, and B. C. Vemuri, "Shape modeling with front propogation: a level set approach", "IEEE Trans. Pattern Anal. Mach. Intell., vol. 17, (1995), pp. 158-175.

[10] Y. Wei, G. Shen and J.-j. Li, "A Fully Automatic Method for Lung Parenchyma Segmentation and Repairing, Journal of Digital Imaging”, vol. 26, no. 3, (2013) June, pp. 483-495.

[11] X. Zhou a,*, T. Hayashi a, T. Hara a, H. Fujita a, R. Yokoyama b, T. Kiryu b and H. Hoshi b, "Automatic segmentation and recognition of anatomical lung structures from high-resolution chest CT images", Computerized Medical Imaging and Graphics", vol. 30, (2006), pp. 299-313.

[12] Digital Image Processing with MATLAB, Gonzalez /woods/Eddins Pearson Education Korea, (2004).

\section{Authors}

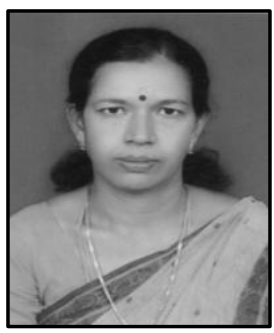

K. Thanammal, she received the M. Sc. (Computer Science) degree from Bharathidasan University, Tiruchirappalli in 1992, M.Phil (Computer Science) degree from Manonmaniam Sundaranar University, Tirunelveli in 1999 and M. Tech. (Computer Science) degree from Vinayaka Mission University, Salem in 2007.She is working as an Associate Professor in Computer Science Department at S.T.Hindu College, Nagercoil since 1992. She has 22 years of teaching experience. She has presented and published 6 papers in National and International Conferences and Journals. Her current research interest focuses on Image Processing, Neural Networks.

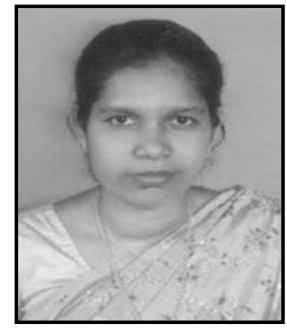

Jayasudha J. S., she received the B.E (Computer Science) degree from R.V.S. College of Engineering, Dindigul in 1994, M.E (Computer Science) degree from National Institute of Technology, Tiruchirappalli in 2002 and Ph.D (Computer Science) degree from Kerala University in 2008. She is working as Asst. Professor and Head in the Department of computer Science \& Engineering, Sree Chitra Thirunal College of Engineering, Thiruvananthapuram, India. She has 16 years of teaching experience. She has presented and published many papers in National and in International Conferences and Journals. Her current research interest focuses on Computer Networks, Image Processing, and Neural Networks. 